\title{
El perfil pedagógico de los MOOC a partir de un estudio exploratorio
}

\author{
The pedagogic profile of the MOOC from an exploratory study
}

\author{
Manuela Raposo-Rivas, ${ }^{a}$ José Sarmiento Campos ${ }^{a b}$ María Martínez-Figueira ${ }^{c}$
}

${ }^{\text {a}}$ Facultad de Ciencias de la Educación, Universidade de Vigo

Telf.: (34) 988387164. Correo electrónico: mraposo@uvigo.es

'Telf.: (34) 988387304 Correo electrónico: sarmiento@uvigo.es

${ }^{c}$ Facultad de Ciencias de la Educación y del Deporte, Universidade de Vigo

Telf.: (34) 986801773. Correo electrónico: esthermf@uvigo.es

\section{RESUMEN}

Los MOOC están revolucionando la enseñanza y el aprendizaje del siglo XXI. Pueden ser de distintos tipos, atendiendo a criterios muy diversos, pero falta caracterizar su pedagogía. Para establecer un perfil pedagógico de los MOOC, se estudian 117 cursos de habla hispana, ubicados en 10 plataformas diferentes, con un diseño mixto de tipo exploratorio secuencial. La información obtenida se analiza por conglomerados en dos fases con los paquetes estadísticos SPSS y Weka. Los resultados muestran dos perfiles: MOOC cerrados y rígidos frente a abiertos y flexibles; e identifican el componente pedagógico que puede clasificarlos, siendo las "horas de dedicación" lo que mejor discrimina a los estudiados.

Palabras clave: cursos online masivos abiertos, pedagogía, análisis de conglomerados.

\section{ABSTRACT}

MOOCs are revolutionizing XXI century teaching and learning. There can be different types according to diverse criteria, but they lack a pedagogical profile. To establish an educational profile of the MOOC, 117 Spanishlanguage courses, located in 10 different platforms, are studied. They are studied using a sequential mixed exploratory research. With the information obtained, an analysis of conglomerates is carried out in two phases using the statistical packages SPSS and Weka. The results show two profiles of courses: closed and rigid MOOCs versus open and flexible MOOCs. They also identify the pedagogical component that can classify a MOOC course. The variable "hours of dedication" best discriminates the courses studied.

Key words: Massive Online Open Courses, pedagogy, cluster analysis. 


\section{A MODO DE INTRODUCCIÓN: TIPOS Y TENDENCIAS PEDAGÓGICAS DE LOS MOOC}

En la actualidad, los MOOC, del acrónimo inglés de Massive Online Open Courses o su correspondiente español COMA, constituyen un verdadero hito pedagógico cuyo nacimiento está apoyado en algo positivo, democrático y universal. Los escenarios formativos toman un nuevo formato que aúnan gratuidad, masividad y ubicuidad (Boxall, 2012; Cormier \& Siemens, 2010). Su rápido desarrollo, junto con el aumento exponencial de la literatura científica y de divulgación (Liyanagunawardena, Adams \& Williams, 2013; Martínez, Rodríguez \& García, 2014; Yuan \& Powell, 2013) así como la celebración de encuentros internacionales, monográficos de publicaciones periódicas e informes de instituciones y organismos (entre otros, Haggar, 2013; Oliver et al., 2014; SCOPEO, 2013), dibujan un nuevo panorama y plantean nuevos retos a la enseñanza y el aprendizaje a finales de la primera década del siglo XXI.

Por su filosofía, estos cursos se extienden a nivel mundial y son un claro ejemplo de disrupción (Anderson \& McGreal, 2012; Conole, 2013; Vázquez-Cano, López \& Sarasola, 2013), solo si se verifica que son tomados como experimentos para poner a prueba nuevas metodologías, nuevas tecnologías y nuevas formas de organizar la educación (Pernías \& Lujan-Mora, 2013). Sin embargo, no hay razón para pensar que son menos eficaces que otras experiencias de aprendizaje en línea, ya que los MOOC pueden tener una base pedagógica sólida (Glance, Forsey \& Riley, 2013).

Sin lugar a dudas, una de las piezas clave e imprescindible para la puesta en marcha de un MOOC es su diseño pedagógico en la medida en que orienta, organiza, estructura, sistematiza, explicita y publicita la acción formativa que se lleva a cabo (Raposo, 2014). En este diseño son cuatro los principios que, según Downes (2013), deben ser considerados: autonomía, diversidad, apertura e interactividad, ya que son claves a la hora de plantear actividades de aprendizaje, materiales y una estructura de participación que aporte valor real, pues será y dependerá de la acción de los participantes. Conole (2013) manifiesta que los cursos pedagógicamente eficaces dan lugar a una experiencia de aprendizaje más rica y aseguran su garantía de calidad.

Así, el planteamiento y desarrollo de estos cursos pivota sobre los estudiantes y sus procesos de aprendizaje, procurando la co-construcción de un aprendizaje autónomo, autorregulado, rizomático (Sharples et al., 2012), situado (Brown, Collins \& Duguid, 1989) y colaborativo. Se debe tener en cuenta, que el reto del aprendizaje colaborativo mediado por entornos virtuales, como en este caso son los MOOC, consiste en encontrar formas de organizar las tareas y de configurar los recursos que lleven a los participantes a modificar su punto de vista mediante el desarrollo de habilidades y competencias con el fin de alcanzar compromisos y conseguir los objetivos compartidos (Escofet \& Marimon, 2012), Así es como los recursos utilizados adquieren un importante papel.

Las TIC por sí solas no potencian nuevas formas de aprender en equipo, pero sí que son transformadoras de las relaciones humanas. Las tecnologías pueden facilitar unas condiciones adecuadas para la colaboración eficaz si refuerzan la dimensión social de la educación. El aprendizaje colaborativo es posible en un entorno social y de relación que priorice la comunicación y el intercambio, y es en este contexto que las telecomunicaciones facilitan algunas características de este entorno de aprendizaje (Escofet \& Marimon, 2012). 
Para ello, en los MOOC habitualmente se plantea el uso de herramientas colaborativas y comunicativas propias de la web 2.0 (blogs, foros, etcétera), mientras que los medios de formato escrito tienen una presencia muy baja, siendo el audiovisual el formato de divulgación preferido (Vázquez-Cano, 2013), quizás por su carácter más dinámico, ameno y visual. En esta línea, Letón, Luque, Monales y García (2013) junto con Montero y Viñuales (2013), sugieren que un MOOC debe asentarse en un modelo de diseño curricular compuesto por unidades didácticas constituidas por videos donde el profesorado apenas intervenga.

De esta forma, estamos ante un formato que promueve activamente la auto-organización, la conectividad, la diversidad y el control descentralizado de los procesos de enseñanzaaprendizaje (Baggaley, 2014). Se percibe cierta tendencia al conectivismo, que según Siemens (2005) supera al conductismo, cognitivismo y constructivismo, resaltando la importancia de la interacción entre todos los participantes en el proceso de aprendizaje, pero añade un matiz en el que profesorado y estudiantes participan como nodos de información, en un proceso asistido por las tecnologías y las conexiones de información con las que se genera el aprendizaje. De hecho, ya Strijbos, Martens y Jochems (2004) abogaban por un modelo que comunique el tipo de interacción esperada, y que clarifique la relación entre el resultado de la tarea y la interacción grupal, teniendo en cuenta seis aspectos fundamentales en el diseño de aprendizaje en grupo basado en el uso del ordenador: (1) determinar los objetivos de aprendizaje, (2) determinar la interacción esperada, (3) seleccionar el tipo de tarea según los objetivos e interacción, (4) determinar cuánta estructuración se necesita según objetivos, interacción y tareas, (5) determinar el tamaño del grupo, y (6) determinar cómo el soporte informático es la mejor opción para apoyar el aprendizaje y la interacción previstos.

Sin embargo, dado el carácter abierto y masivo de los MOOC existe una inmensa diversidad de usuarios con variedad de intereses y motivaciones, enfoques y estilos de aprendizaje, lo que hace que uno de los retos más difíciles de estos cursos sea proporcionar experiencias de aprendizaje auténtico.

Tecnológicamente, los MOOC se asientan en variadas y diversas plataformas con orígenes y enfoques diferentes, lo que ha dado lugar, por ejemplo, a que se hable de xMOOC y cMOOC como los dos principales tipos (Cabero, Llorente \& Vázquez, 2014; Downes, 2013; Hill, 2012; Siemens, 2012; Vázquez et al., 2013) o bien, un modelo híbrido de ellos considerando tres categorías básicas (Martí, 2012; Pernías \& Lujan-Mora, 2013; Vázquez-Cano, 2013):

- MOOC basados en el aprendizaje distribuido en red, fundamentado en la teoría conectivista y su modelo de aprendizaje (Siemens, 2005).

- MOOC basados en tareas, según las habilidades del alumnado en la resolución de determinados tipos de trabajo (Cornier \& Siemens, 2010).

- $\quad$ MOOC basados en contenidos, se fundamentan en un modelo de evaluación parecido a las clases tradicionales.

Por su parte, Clark (2013) identifica hasta ocho tipos:

- TransferMOOCs: los cursos existentes en las Universidades de e-learning se transfieren a una plataforma MOOC.

- MadeMOOCs: incorporan elementos de video, hacen hincapié en la calidad de la creación de tareas, potencian el trabajo entre iguales y la coevaluación. 
- SynchMOOCs: los cursos presentan fechas específicas de comienzo y finalización, así como de realización de las evaluaciones.

- AsynchMOOCs: sin fechas límites.

- AdaptiveMOOCs: utilizan algoritmos adaptativos para presentar experiencias de aprendizaje personalizadas, basadas en la evaluación dinámica y la recopilación de datos del curso.

- GroupMOOCs: elaborados para grupos específicos.

- ConnectivistMOOCS: los propuestos por Siemens (2012).

- MiniMOOCS: de corto número de contenidos y plazos de desarrollo.

Atendiendo a un conjunto de doce dimensiones, Conole (2013) clasifica los MOOC según el grado de apertura, la escala de participación (masificación), la cantidad de uso de multimedia, la densidad de comunicación, el grado de colaboración que incluye, el itinerario de aprendizaje, el nivel de aseguramiento de la calidad, el grado en que alienta a la reflexión, la acreditación, el grado de formalidad, autonomía y diversidad.

No obstante, creemos que en un curso online masivo abierto, tecnología y pedagogía, plataformas y diseño instruccional están íntimamente ligados, hasta el punto que Roig, Mengual-Andrés y Suárez (2014) no encuentran una diferencia significativa entre la calidad pedagógica de los MOOC y la plataforma que los contiene. Sin embargo, aunque la plataforma ofrece un soporte tecnológico necesario, la propuesta de MOOC debería tener su propia autonomía (Raposo-Rivas, Martínez-Figueira \& Sarmiento, 2015). Dichos autores han podido comprobar que el diseño pedagógico de los cursos analizados está determinado por la plataforma; se repite una y otra vez (tantas como plataformas estudiadas) el mismo patrón que conforman, entre otros, las actividades y materiales, modalidades de aprendizaje, propuestas de evaluación, nivel de interactividad, acceso y certificación; es decir, la plataforma constriñe y encorseta los cursos online, si bien, en algunas de ellas hay cierta flexibilidad, con fronteras más "líquidas" entre los diferentes indicadores pedagógicos.

La propuesta que aquí se presenta analiza los componentes pedagógicos que poseen 117 MOOC para intentar identificar perfiles a partir de dichos cursos, no con criterios tecnológicos asentados en las plataformas, sino con criterios pedagógicos basados en el diseño instructivo de la formación. Así, son dos las finalidades de este trabajo:

- $\quad$ Presentar las tendencias que siguen los perfiles de MOOC en lengua española basados en sus componentes pedagógicos.

- Comprobar si existe algún componente común a los MOOC, independiente de las plataformas que lo soportan.

\section{DISEÑO DE LA INVESTIGACIÓN}

Los datos analizados forman parte de una investigación más amplia (Tabla 1), que procura evitar el excesivo énfasis empirista basado en las relaciones lineales causa-efecto entre variables y experimentar efectos dinámicos entre variables (Galán, Ruíz-Corbella \& Sánchez, 2014). Sus objetivos son:

Analizar la oferta de MOOC disponible en habla hispana durante un período determinado para poder establecer un perfil de componentes pedagógicos. 
- Validar un instrumento que pueda orientar el diseño pedagógico de los MOOC.

- Determinar si los componentes pedagógicos de los MOOC están condicionados por las plataformas.

- Categorizar los cursos MOOC según los componentes pedagógicos presentes en su diseño.

- Identificar aquel o aquellos componente-s pedagógico-s que le pueden dar identidad al curso MOOC.

Tabla 1. Síntesis de la investigación

\begin{tabular}{|c|c|}
\hline Elementos & Descripción \\
\hline Metodología & $\begin{array}{l}\text { Estudio descriptivo con un diseño mixto (DEXPLOS) de tipo exploratorio se- } \\
\text { cuencial (Creswell et al., 2008; Hernández, Fernández y Baptista, 2010), con una } \\
\text { fase inicial de recolección y análisis de datos cualitativos seguida de otra donde } \\
\text { se recaban y analizan datos cuantitativos. }\end{array}$ \\
\hline $\begin{array}{l}\text { Tipo de mues- } \\
\text { treo y criterios } \\
\text { de selección de } \\
\text { la muestra }\end{array}$ & $\begin{array}{l}\text { Muestreo criterial (McMillan y Schumacher, 2005) y secuencial para métodos } \\
\text { mixtos (Hernández et al., 2010). } \\
\text { Criterios de selección: catalogados en el repositorio www.MOOC.es; idioma es- } \\
\text { pañol; información disponible sin tener que matricularse en la plataforma; pro- } \\
\text { porcionan un nivel mínimo de información en el instrumento de recogida de datos } \\
\text { (INdiMOOC-EdI). }\end{array}$ \\
\hline Muestra & $\begin{array}{l}\text { Muestra cualitativa: } 104 \text { cursos }(81.25 \%) \text { de diferentes áreas de conocimiento } \\
\text { pertenecientes a } 8 \text { plataformas distintas. }\end{array}$ \\
\hline (Ver Figura 1) & $\begin{array}{l}\text { Muestra cuantitativa: } 117 \text { cursos de diferentes áreas de conocimiento ubicados en } \\
10 \text { plataformas diferentes. }\end{array}$ \\
\hline Instrumento & $\begin{array}{l}\text { INdiMOOC-EdI (Instrumento de Indicadores Educativos e Interactivos en un } \\
\text { MOOC) organizado en cuatro componentes: datos de identificación, aspectos } \\
\text { descriptivos, formativos e interactivos (Raposo-Rivas et al., 2015). }\end{array}$ \\
\hline $\begin{array}{l}\text { Validez y fiabi- } \\
\text { lidad }\end{array}$ & 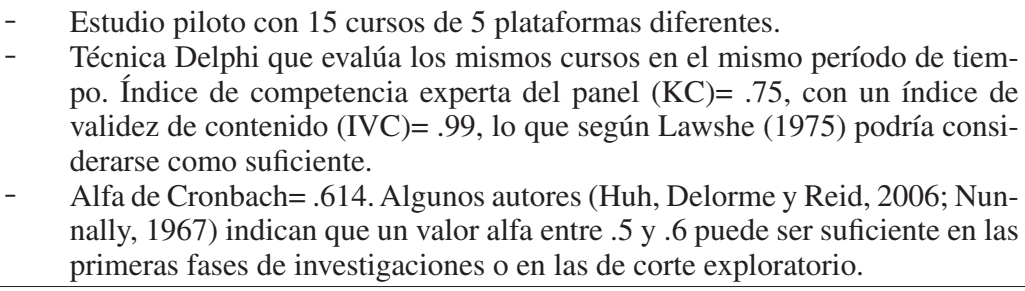 \\
\hline $\begin{array}{l}\text { Análisis de } \\
\text { datos }\end{array}$ & $\begin{array}{ll}- & \text { Análisis de contenido. } \\
\text { - } & \text { Algoritmos propios de la minería de datos }{ }^{1} \text { : de clasificación y de evaluación. } \\
\text { - } & \text { Análisis descriptivo. } \\
\text { - } & \text { Análisis de componentes principales categóricos. } \\
& \text { Técnicas de análisis de mixtura (García, 2011). }\end{array}$ \\
\hline $\begin{array}{l}\text { Software de } \\
\text { análisis }\end{array}$ & $\begin{array}{l}\text { Datos cualitativos: AQUAD6. } \\
\text { Datos cuantitativos: SPSS } 18.0 \text { y Weka (Hall et al., 2009). }\end{array}$ \\
\hline
\end{tabular}

${ }^{1}$ Las técnicas de minería de datos tienen como objetivo la extracción de información relevante de grandes bases de datos empleando para ello algoritmos o técnicas que tratan de localizar información no trivial (Castro \& Lizasoain, 2012). 
Para ello se han analizado 117 cursos de distintas áreas de conocimiento accesibles en marzo de 2014. En la Figura 1 se muestra su distribución en las diferentes plataformas: el $100 \%$ de los MOOC disponibles en ese periodo de tiempo en AbiertaUGR, UCAM y UNED-COMA. Del resto de plataformas, se puede indicar que de MiriadaX y UNIMOOC se ha considerado también un porcentaje muy elevado de la población (98\% y 90\%, respectivamente), seguido de Coursera (88\%), UnX (83\%) y UPVX (43\%).

Figura 1. Muestra productora de datos

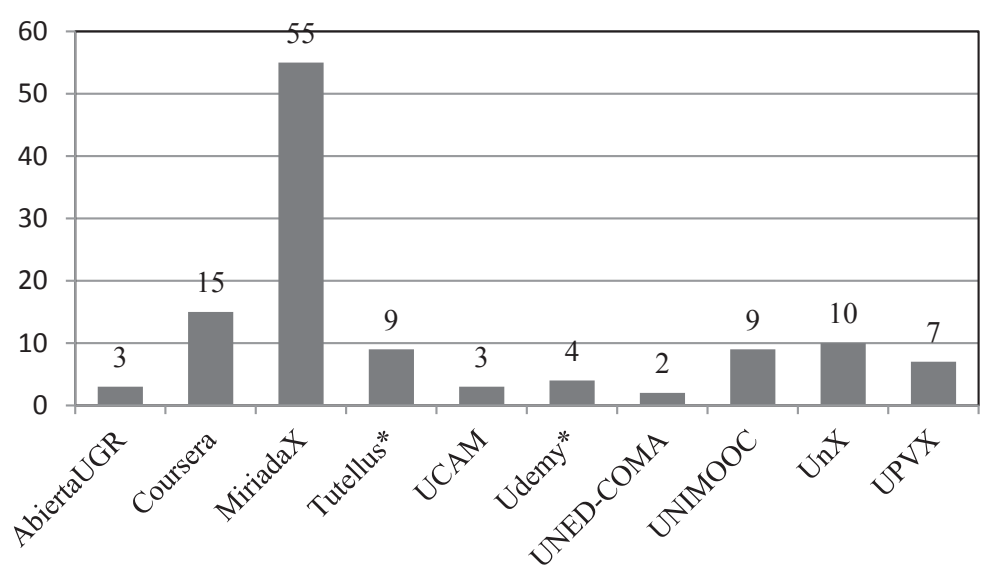

Fuente: elaboración propia.

Las plataformas Tutellus y Udemy poseen una representación mínima (.22\% y $2.9 \%$, respectivamente) ya que en ellas se localizan cursos que no pueden encuadrarse dentro de la categoría MOOC (Raposo-Rivas et al., 2015) como son conferencias, videoconferencias o clases magistrales en soporte audiovisual, que sugieren ser un reciclaje de materiales de diferentes procedencias pertenecientes al repositorio audiovisual de una institución para ahora ser presentados como cursos masivos. Además, aportan escasa información en el instrumento de investigación sin inscribirse en ellas, por ejemplo, información redundante sobre el manejo de la plataforma, certificaciones, etc. Se observa una saturación semántica tal que, con independencia del curso, los datos proporcionados son los mismos.

Así, estas dos plataformas no se tienen en cuenta para la determinación de la muestra utilizada en el análisis cualitativo de los datos. Con ello se puede confirmar que los 104 cursos restantes significan el $81.25 \%$ de la población.

Se utiliza como instrumento, INdiMOOC-EdI (Raposo-Rivas et al., 2015), formado por 27 cuestiones medidas en varias escalas organizadas en cuatro apartados: datos de identificación, aspectos descriptivos, formativos e interactivos.

En esta propuesta se trabaja con los datos obtenidos a partir de 23 componentes pedagógicos que, en consonancia con los objetivos de la investigación se analizan con los paquetes estadísticos SPSS 18.0 y Weka (Hall et al., 2009). Se utiliza la técnica estadística de conglomerados en dos fases o bietápica que ayuda a determinar el número ideal de 
conglomerados a partir de los datos recogidos, lo que nos acerca al número de perfiles de MOOC que se pueden identificar. En segundo lugar, este análisis agrupa dichos datos en su conglomerado correspondiente, creando una nueva variable "número de conglomerado en dos fases".

Con la finalidad de confirmar y complementar los resultados de la técnica anterior se emplea otro algoritmo de agrupamiento, esta vez implementado en el paquete estadístico Weka. Una variante del algoritmo K-medias que utiliza la moda en lugar de la media como medida de tendencia central. Finalmente, se ha aplicado el algoritmo de clasificación mediante segmentación jerárquica CHAID (Chi_square Automatic Interaction Detection) a los datos estableciendo como variable de clasificación "número de conglomerado en dos fases".

\section{RESULTADOS Y DISCUSIÓN}

Se presentan los resultados obtenidos utilizando una técnica de conglomerado en dos fases (bietápica y K-modas) junto con el algoritmo CHAID, para dirimir si se identifica una tendencia en los componentes pedagógicos de los MOOC que permita establecer perfiles y si hay algún componente discriminante en ellos.

\section{1. ¿SE IDENTIFICA UNA TENDENCIA EN LOS COMPONENTES PEDAGÓGICOS DE LOS MOOC QUE PERMITA HABLAR DE PERFILES?}

El resultado de la técnica de conglomerado bietápica establece como 2 el número ideal de conglomerados (Tabla 2), ya que es con este número de agrupamientos cuando la relación entre el Criterio Bayesiano de Schwarz (BIC) y su tasa de cambios es mayor (Pérez, 2005).

Tabla 2. Selección del número de conglomerados mediante BIC

\begin{tabular}{|c|c|c|c|c|}
\hline $\begin{array}{c}\text { Número de } \\
\text { conglomerados }\end{array}$ & $\begin{array}{c}\text { Criterio } \\
\text { BIC }\end{array}$ & $\begin{array}{c}\text { Cambio } \\
\text { en BIC }\end{array}$ & $\begin{array}{c}\text { Razón de cambios } \\
\text { en BIC }\end{array}$ & $\begin{array}{c}\text { Razón de medidas } \\
\text { de distancia }\end{array}$ \\
\hline 1 & 5070.645 & & & \\
\hline $\mathbf{2}$ & $\mathbf{4 2 1 2 . 5 5 0}$ & $\mathbf{- 8 5 8 . 0 9 5}$ & $\mathbf{1 . 0 0 0}$ & $\mathbf{2 . 5 3 6}$ \\
\hline 3 & 4032.893 & -179.657 & .209 & 1.377 \\
\hline 4 & 3974.042 & -58.851 & .069 & 1.147 \\
\hline 5 & 3956.392 & -17.650 & .021 & 1.383 \\
\hline
\end{tabular}

Fuente: elaboración propia.

En primer lugar, con el análisis bietápico, dichos conglomerados agrupan al $60 \%$ $(\mathrm{n}=70)$ y $40 \%(\mathrm{n}=47)$ de los cursos respectivamente. En segundo lugar, cuando se aplica el algoritmo K-modas a los datos se obtiene una distribución de los cursos de $67 \%$ (n=78) frente al $33 \%(n=39)$. 
Así, la implementación de las dos técnicas de conglomerado muestra que el porcentaje de coincidencia alcanza el 93\% (Tabla 3 ).

Tabla 3. Matriz de confusión perteneciente al análisis de conglomerados en dos fases

\begin{tabular}{|l|c|c|c|}
\hline Algoritmo K-modas & Conglomerado 1 & Conglomerado 2 & Totales \\
\hline Conglomerado 1 & $70(60 \%)$ & $8(7 \%)$ & $78(67 \%)$ \\
\hline Conglomerado 2 & $0(0 \%)$ & $39(33 \%)$ & $39(33 \%)$ \\
\hline Totales & $70(60 \%)$ & $47(40 \%)$ & $117(100 \%)$ \\
\hline
\end{tabular}

Fuente: elaboración propia.

En base a los resultados obtenidos mediante las dos técnicas anteriores podemos distribuir las respuestas más habituales según el conglomerado de pertenencia a cada uno de los 23 componentes pedagógicos analizados (tablas 4,5 y 6 ).

Tabla 4. Componentes de los datos de identificación de los MOOC pertenecientes a cada conglomerado

\begin{tabular}{|l|l|l|}
\hline \multicolumn{1}{|c|}{ Respuesta principal GRUPO 1 } & \multicolumn{1}{|c|}{ COMPONENTE } & \multicolumn{1}{|c|}{ Respuesta principal GRUPO 2 } \\
\hline Coursera; MiriadaX & Plataforma & El resto \\
\hline Universidad; Universidad-empresa & Institución & $\begin{array}{l}\text { Empresa privada; Iniciativa parti- } \\
\text { cular }\end{array}$ \\
\hline $\begin{array}{l}\text { Artes y humanidades; Ciencias de } \\
\text { la salud; Científico }\end{array}$ & Ámbito & --- \\
\hline Entre dos y cinco & Equipo docente & --- \\
\hline Se accede indirectamente & Perfiles equipo docente & Se accede directamente; No hay \\
\hline $\begin{array}{l}\text { Abierta en un período determina- } \\
\text { do; Cerrado }\end{array}$ & Inscripción & Abierta permanentemente \\
\hline No aparece & Cursos relacionados & Varios \\
\hline--- & Título & --- \\
\hline
\end{tabular}

Fuente: elaboración propia.

Según los datos de identificación del instrumento INdiMOOC-EdI, el grupo 1 tiene un perfil más señalado, con componentes claramente definidos. Forman parte de él aquellos MOOC de las plataformas Coursera y MiriadaX, lo que significa fundamentalmente 
instituciones universitarias o que vinculan la universidad y la empresa, centrados en los ámbitos de Ciencias de la Salud y Científico junto con Artes y Humanidades; con un equipo docente pequeño que presenta un determinado perfil profesional y la inscripción limitada a un período determinado.

Configuran el grupo 2 los MOOC pertenecientes a las plataformas Abierta UGR, Tutellus, Ucam, Udemy, UnedComa, UniMOOC, UnX, UPVX, gestionados desde empresas privadas o iniciativas particulares, un equipo docente del que no siempre se detalla su perfil, una inscripción abierta permanentemente y con sugerencias de otros cursos relacionados.

Al mismo tiempo, el título no es un elemento discriminante de los grupos, tampoco lo son la pertenencia de los cursos multidisciplinares o propios del área Jurídico-Social y Tecnológico y los MOOC gestionados por un docente o más de cinco.

Tabla 5. Componentes de los aspectos descriptivos de los MOOC pertenecientes a cada conglomerado

\begin{tabular}{|l|l|l|}
\hline Respuesta principal GRUPO 1 & COMPONENTE & Respuesta principal GRUPO 2 \\
\hline No se explicita & Importancia para el público & Sí se explicita \\
\hline No se explicita & Destinatarios & $\begin{array}{l}\text { Público en general (interesados } \\
\text { en la temática) }\end{array}$ \\
\hline Sí hay & Prerrequisitos & --- \\
\hline Acotado & Duración del curso & Indefinido; No aparece \\
\hline Más de cinco & Duración semanas & Hasta cinco \\
\hline Acotado (horas x semana) & Dedicación & No se especifica \\
\hline Hasta tres; más de cuatro & Dedicación horas & No se especifica \\
\hline $\begin{array}{l}\text { Se refiere al contenido del } \\
\text { curso; otra }\end{array}$ & Introducción & --- \\
\hline Hay & Video introductorio & No hay \\
\hline No hay & Objetivos & Hay \\
\hline Orientado desde la plataforma & Funcionamiento del sistema & $\begin{array}{l}\text { No se especifica, orientado des- } \\
\text { de el curso }\end{array}$ \\
\hline
\end{tabular}

Fuente: elaboración propia.

En relación con los aspectos descriptivos del instrumento INdiMOOC-EdI, la diferencia de perfil entre los conglomerados no es tan acusada. En los MOOC del grupo 1 se indican prerrequisitos de acceso, la duración necesaria está claramente acotada en semanas (habitualmente más de cinco); la dedicación exigida al usuario en horas semanales (menos de tres o más de cuatro), posee un video introductorio y una introducción referida 
al contenido del curso o más variada y el funcionamiento del sistema se orienta desde la plataforma. Por el contrario, no se explicitan ni la importancia del curso para el público, ni los destinatarios ni los objetivos del mismo.

En el grupo 2, estarían aquellos cursos que indican los objetivos, la importancia para el público y los destinatarios con una duración indefinida o inexistente. En estos MOOC no se contempla la dedicación necesaria ni el video introductorio y el funcionamiento del sistema está orientado desde el curso o bien no se recoge. En este grupo, los prerrequisitos y la introducción no son elementos discriminantes.

Tabla 6. Componentes de los aspectos formativos de los MOOC pertenecientes a cada conglomerado

\begin{tabular}{|l|l|l|}
\hline Respuesta principal GRUPO 1 & COMPONENTE & Respuesta principal GRUPO 2 \\
\hline Por semanas (cerrado) & Plan de trabajo & No se especifica \\
\hline Seis o siete & Módulos & --- \\
\hline Ambas & Certificación & Gratuita, de pago \\
\hline Mixta & Acreditación & $\begin{array}{l}\text { Medallas, insignias, credenciales, } \\
\text { certificados }\end{array}$ \\
\hline
\end{tabular}

Fuente: elaboración propia.

Por último, en relación con los aspectos formativos del instrumento INdiMOOC-EdI, de nuevo el grupo 1 presenta un perfil más acusado. Así en este se detalla el plan de trabajo concretado por semanas, el número de módulos (habitualmente entre seis o siete), el tipo de obtención de la certificación (gratuita y de pago) junto con una acreditación mixta.

Por su parte, los MOOC del grupo 2 poseen una certificación gratuita o de pago, junto con una acreditación por medallas, insignias, credenciales o certificados. El plan de trabajo no se detalla y el número de módulos es un elemento no discriminante.

\section{2. ¿QUÉ COMPONENTE O COMPONENTES PEDAGÓGICOS PUEDEN CLASIFICAR A UN MOOC CON INDEPENDENCIA DE LA PLATAFORMA?}

Para responder a esta cuestión se ha implementado el algoritmo CHAID a los cursos pertenecientes a los dos grupos definidos con anterioridad, que representa el árbol de clasificación resultante (Figura 2). La variable "dedicación horas" fue la única considerada por dicho algoritmo con un chi-cuadrado $=38.778$ y significatividad $=0$. Así, se establecen dos nodos, el primero se refiere a los 61 cursos $(52.1 \%)$ que no contemplan la dedicación en horas o esta es de cuatro horas. El segundo nodo agrupa a los 56 MOOC (47.9\%) con dedicación de hasta tres horas o más de cuatro. Se observa que mientras que el primer nodo se balancea hacia el grupo 2 establecido en los conglomerados anteriores (41, 61.67.2\%), el segundo nodo concentra la mayoría de cursos pertenecientes al grupo 1 (50, 89.3\%). 
Figura 2. Representación gráfica del resultado del algoritmo CHAID

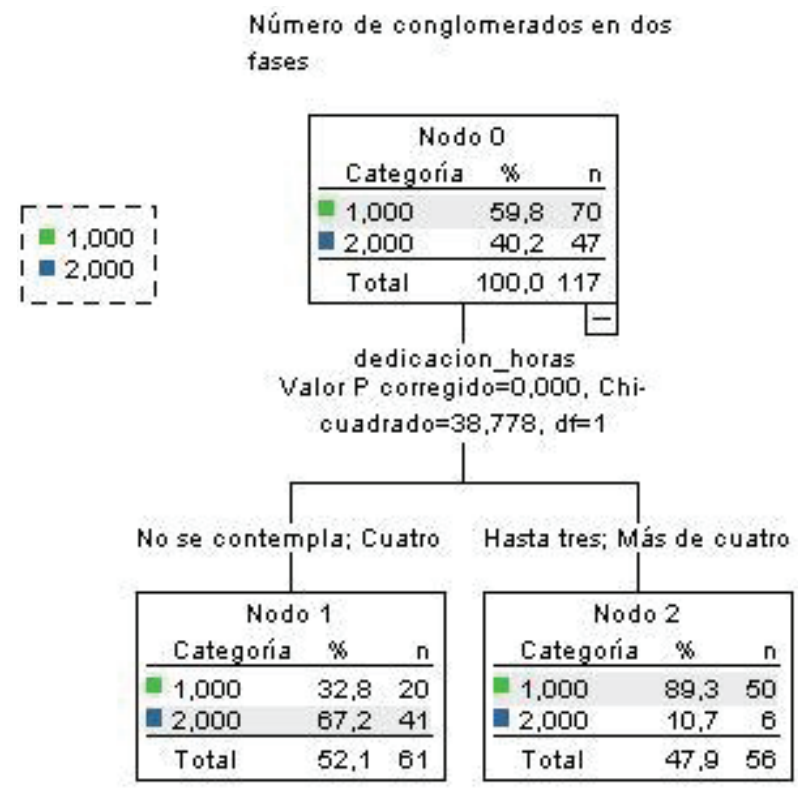

Fuente: elaboración propia.

En la siguiente tabla se puede observar que la variable número de horas de dedicación al curso discrimina razonablemente bien la pertenencia a uno u otro grupo en la totalidad de MOOC analizados (77.8\% de éxito, $\mathrm{n}=91)$. Al mismo tiempo, el grupo 2 es el mejor clasificado con un $87.2 \%$ de porcentaje correcto.

Tabla 7. Matriz de confusión del algoritmo CHAID

\begin{tabular}{|l|c|c|c|}
\hline Observado & \multicolumn{3}{|c|}{ Pronosticado } \\
\hline & 1 & 2 & Porcentaje correcto \\
\hline 1 & $\mathbf{5 0}$ & 20 & $71.4 \%$ \\
\hline 2 & 6 & $\mathbf{4 1}$ & $87.2 \%$ \\
\hline Porcentaje global & $47.9 \%$ & $52.1 \%$ & $77.8 \%$ \\
\hline
\end{tabular}

Nota. Métodos de crecimiento: CHAID. Variable dependiente: número de conglomerados en dos fases.

Fuente: elaboración propia. 


\section{CONCLUSIONES}

A pesar de que en estos últimos años han proliferado diferentes tipos de trabajos en torno a los MOOC, hemos podido comprobar que la atención de estos se ha dirigido más hacia las características de las plataformas donde se alojan y menos a los aspectos pedagógicos de los cursos MOOC. Analizando este contenido, Rosselle, Caron y Heutt (2014) se aventuran a ofrecer un avance de tipologías de cursos en función de los elementos que incluyen o Sarabia (2016) aporta un análisis en base a las características de los cursos MOOC de reciente implantación en las universidades españolas, para terminar aportando una serie de descriptores y dimensiones que caracterizan estos cursos. En este trabajo se han estudiado también los elementos que incluyen una muestra de 117 MOOC de habla hispana con la finalidad de identificar una determinada tipología y perfil pedagógico en ellos.

A partir de este propósito, los resultados obtenidos muestran los perfiles de MOOC estudiados a partir de los componentes pedagógicos identificados con el instrumento de análisis INdiMOOC-EdI. Esto quedó dibujado en las tablas 4, 5 y 6, lo que permite concluir que, en el período estudiado, existen dos perfiles diferenciados definidos por datos de identificación, aspectos descriptivos y formativos del curso: MOOC rígidos y cerrados (grupo 1), frente a MOOC flexibles y abiertos (grupo 2).

La naturaleza del primer perfil es de carácter más rígido y cerrado, con 22 componentes pedagógicos claramente definidos de los 23 estudiados (95.6\%). En él se concentran 70 cursos $(59.83 \%)$ de carácter universitario vinculados a las plataformas Coursera y MiriadaX $(\mathrm{n}=15$ y $n=55$, respectivamente) centrados en los ámbitos de Ciencias de la Salud, Científico junto con Artes y Humanidades; con un equipo docente pequeño con un determinado perfil profesional y la inscripción limitada a un período determinado. En estos cursos, como dicen Vidal y Camarena (2014), el docente, como figura física, es corporalmente difusa, pero su papel facilitador del proceso enseñanza-aprendizaje es clave; en él recae la responsabilidad de reforzar la participación e interacción de y entre estudiantes, y con la plataforma, actúa como protagonista, mediador y facilitador de tal proceso.

En este primer perfil, también se especifican los prerrequisitos de acceso, la duración necesaria acotada en semanas; la dedicación exigida al usuario en horas semanales; un video introductorio y una introducción referida al contenido del curso o más variada; se detalla el plan de trabajo concretado por semanas, el número de módulos; el tipo de certificación y una acreditación mixta. El funcionamiento del sistema se orienta desde la plataforma.

Por el contrario, la naturaleza del segundo perfil es de carácter más flexible y abierto, con 17 componentes pedagógicos identificados de los 23 estudiados (73.9\%). En este perfil se localizan los restantes $44 \mathrm{MOOC}(37.61 \%)$ que se ubican en 8 plataformas diferentes (Abierta UGR, Tutellus, Ucam, Udemy, UnedComa, UniMOOC, UnX, UPVX), gestionados desde empresas privadas o iniciativas particulares, una inscripción abierta permanentemente; indican los objetivos, la importancia para el público y los destinatarios; poseen una certificación gratuita o de pago, junto con una acreditación por medallas, insignias, credenciales o certificados; recomiendan otros cursos relacionados y el funcionamiento del sistema está orientado desde el curso. Debemos tener en cuenta que "la existencia de unos objetivos de aprendizaje explícitamente planteados tiene relación con una alta puntuación en la calidad pedagógica de los MOOC” (Roig et al., 2014, p. 37). Además, según Vázquez-Cano (2013) y Zapata-Ros (2013a), la información previa al curso que se proporciona, los enfoques pedagógicos, el nivel de compromiso de los estudiantes, 
el papel del equipo docente, la disponibilidad y el nivel de interacción, la tipología de recursos, la estructura del curso y el proceso de certificación, entre otros, son aspectos que también se relacionan con la calidad del MOOC.

Por otro lado, la variable "dedicación en horas" se muestra como un componente común a los MOOC, independiente de las plataformas. Es importante resaltar el hecho de que la plataforma de pertenencia no es considerada por el algoritmo CHAID (Figura 2), lo que da cuenta de la relativa independencia del resultado obtenido con respecto a las diez plataformas en donde se alojan los 117 cursos analizados. Al mismo tiempo, esta variable discrimina de forma más efectiva los cursos pertenecientes al grupo 2 que hemos denominado MOOC flexibles y abiertos.

Así, aportamos una nueva perspectiva para considerar a la hora de establecer categorías o hablar de tipologías de MOOC, no tanto desde las plataformas (Cabero et al., 2014; Downes, 2013; Vázquez et al., 2013; Hill, 2012; Siemens, 2012), las teorías de aprendizaje subyacente (Siemens, 2005) o las tareas propuestas (Cornier \& Siemens, 2010), sino desde los componentes pedagógicos disponibles en la carta de presentación de un determinado MOOC. A pesar de que este perfil se ha obtenido en un determinado espacio temporal, hipotetizamos que la distribución de MOOC en flexibles y abiertos frente a MOOC rígidos y cerrados podría mantenerse en el tiempo, llegando incluso a aumentar la distancia entre dichos extremos. Esto que puede ser una limitación, se convierte en la oportunidad de seguir nuevas líneas de estudio, centradas en la mejora del instrumento utilizado y en un seguimiento de las tendencias y los perfiles pedagógicos detectados o de otros posibles emergentes.

Finalmente, los MOOC pueden resultar una útil experiencia de autoaprendizaje en el proceso de formación a lo largo de la vida, acercando la oferta formativa a usuarios o sectores a los que no ha llegado la educación superior más tradicional; sin perder de vista, como indica Aguaded (2013), que pueden ser una oportunidad de aprendizaje excepcional, pero a día de hoy son todavía insuficientes por sí mismos como una experiencia educativa. Medina-Salguero y Aguaded (2013) apuntan sobre estos que "no cambiarán de raíz la formación reglada, pero acabarán influyendo en la manera en que enseñemos en ella, ayudándonos a construir esa universidad del futuro. No serán un modelo alternativo, pero sí será un proceso enriquecedor, complementario y dinamizador" (p. 35).

Con independencia de que el avance y evolución de los MOOC derive, por ejemplo, en Blended MOOC (Lamartina, 2013); en MOOR (Massive Online Open Resources; Méndez, 2013); en POOC (Personal Open Online Courses; Zapata-Ros, 2013b) o en LOOC (Little Open Online Coursers; Medina-Salguero \& Aguaded, 2013), creemos que es positivo la existencia de estas iniciativas de formación ya que abren nuevas posibilidades a la educación, permiten una mayor democratización del conocimiento y complementan las existentes en aras a la mejora de la calidad educativa.

\section{REFERENCIAS BIBLIOGRÁFICAS}

Aguaded, I. (2013). La revolución MOOCs, ¿una nueva educación desde el paradigma tecnológico? Comunicar, 41, 7-8. doi:10.3916/C41-2013-a1

Anderson, T., \& Mcgreal, R. (2012). Disruptive Pedagogies and Technologies in Universities. Education, Technology and Society, 15(4), 380-389. 
Baggaley, J. (2014). MOOC postscript. Distance Education, 35(1), 126-132. doi:10.1080/0158791 9.2013.876142

Boxall, M. (2012). MOOCs: A Massive Opportunity for Higher Education, or Digital Hype? The Guardian Higher Education Network. Recuperado desde http://goo.gl/nS4px2

Brown, J. S., Collins, A., \& Duguid, P. (1989). Situated cognition and the culture of learning. Educational Researcher, 18(1), 32-42.

Cabero, J., Llorente, M. C., \& Vázquez, A. I. (2014). Las tipologías de MOOC: su diseño e implicaciones educativas. Profesorado. Revista de curriculum y formación del profesorado, 18(1), 13-26.

Castro, M., \& Lizasoain, L. (2012). Las técnicas de modelización estadística en la investigación educativa: minería de datos, modelos de ecuaciones estructurales y modelos jerárquicos lineales. Revista Española de Pedagogía, 251, 131-148.

Clark, D. (2013). MOOCs: taxonomy of 8 types of MOOC. Donald Clark Plan B. Recuperado desde http://goo.gl/P4Hb1g

Conole, G. (2013). MOOCs as Disruptive Technologies: Strategies for Enhancing the Learner Experience and Quality of MOOCs. RED, 39, 1-18. Recuperado desde http://www.um.es/ead/ $\mathrm{red} / 39 /$

Cormier, D., \& Siemens, G. (2010). Throught the Open Door: Open Courses as Research, Learning y Engagement. Educase Review, 45(4), 30-39.

Creswell, J. W., Plano, V. L., Gutmann, M. L., \& Hanson, W. E. (2008). Advanced mixed methods research designs. In V. L. Plano, \& J. W. Creswell (Eds.), The mixed methods reader (pp. 161196). Thousand Oaks, CA, USA: Sage.

Downes, S. (2013). The Quality of Massive Open Online Courses by Stephen Downes. Recuperado desde http://goo.gl/vL7G6b

Escofet, A., \& Marimon, M. (2012). Indicadores de análisis de procesos de aprendizaje colaborativo en entornos virtuales de formación universitaria. Enseñanza y Teaching, 30, 85-114.

Galán, A., Ruíz-Corbella, M., \& Sánchez, J.C. (2014). Repensar la investigación educativa: de las relaciones lineales al paradigma de la complejidad. Revista Española de Pedagogía, 258, 281298.

García, A. (2011). Técnicas actuales de estadística aplicada. Madrid: UNED.

Glance, D. G., Forsey, M., \& Riley, M. (2013). The Pedagogical Foundations of Massive Open Online Courses. First Monday, 18(5), 1-10. doi:10.5210/fm.v18i5.4350

Haggard, S. (2013). Massive Open Online Courses and Online Distance Learning: review. GOV.UK Research and Analysis. UK: Universities UK.

Hall, M., Frank, E., Holmes, G., Pfahringer, B., Reutemann, P., \& Witten, I. H. (2009). The WEKA Data Mining Software: An Update. SIGKDD Explorations, 11(1), 10-18.

Hernández, R., Fernández, C., \& Baptista, P. (2010). Metodología de la investigación. Madrid: Pearson.

Hill, P. (2012). Four Barriers that MOOCs must overcome to build a sustainable model e-Literate. Recuperado desde http://goo.gl/GMahGR

Huh, J., Delorme, D. E., \& Reid, L. N. (2006). Perceived Third-Person Effects and Consumer Attitudes on Prevetting and Banning DTC Advertising. Journal of Consumer Affairs, 40(1), 90116. doi:10.1111/j.1745-6606.2006.00047.x

Lamartina, D. (2013). Blended MOOCs: The best of both worlds? Campus Tecnology, 26(12), 20-22.

Lawshe, C. H. (1975). A Quantitative Approach to Content Validity. Personnel Psychology, 28, 563575.

Letón, E., Luque, M., Monales, E. M., \& García, T. (2013) ¿Cómo diseñar un MOOC basado en mini-vídeos docentes modulares? Recuperado desde http://goo.gl/n5AUik

Liyanagunawardena, T., Adams, A., \& Williams, A. (2013). MOOCs: A Systematic Study of the Published Literature 2008-12. The International Review of Research in Open and Distance 
Learning, 14(3), 202-227.

Martí, J. (2012). Tipos de MOOCs. Recuperado desde http://www.xarxatic.com/tipos-de-moocs/

Martínez, F., Rodríguez, M. J., \& García, F. (2014). Evaluación del impacto del término "MOOC" vs. "Elearning" en la literatura científica y de divulgación. Revista de Currículum y Formación del Profesorado, 18(1), 186-201.

Mcmillan, J., \& Schumacher, S. (2005). Investigación educativa. Madrid: Pearson.

Medina-Salguero, R., \& Aguaded, I. (2013). La ayuda pedagógica en los MOOC: un nuevo enfoque en la acción tutorial. @ tic. revista d'innovació educativa 11, 30-39.

Méndez, C. (2013). Diseño e implementación de cursos abiertos masivos en línea (MOOC): expectativas y consideraciones prácticas. RED. Revista de Educación a Distancia, 39,1-19.

Montero, I., \& Viñuales, J. (2013). Difundi y OpenMOOC. Desarrollando un modelo de negocio basado en servicios MOOC y sobre una plataforma de software libre. In SCOPEO (Coord.), MOOC: Estado de la situación actual, posibilidades, retos y futuro. Informe SCOPEO $n^{\circ} 2$ (pp. 140-155). Recuperado desde http://goo.gl/UgZfv7

Nunnally, J. C. (1967). Psychometric theory. New York: McGraw-Hill.

Oliver, M., Hernández-Leo, D., Daza, V., Martín, C., \& Albó, L. (2014). MOOCs en España. Barcelona: Universitat Pompeu Fabra.

Pérez, C. (2005). Métodos estadísticos avanzados con SPSS. Madrid: Thomson.

Pernías, P., \& Luján-Mora, S. (2013). Los MOOC: Orígenes, historia y tipos. Comunicación y Pedagogía, 269-270, 41-47.

Raposo, M. (2014). Orientaciones pedagógicas para los MOOC. Ponencia presentada en III Workshop Internacional sobre Creación de MOOC con anotaciones multimedia. Málaga.

Raposo-Rivas, M., Martínez-Figueira, E., \& Sarmiento, J. A. (2015). Un estudio sobre los componentes pedagógicos de los cursos online masivos. Comunicar, 44, 27-35. doi:10.3916/ C44-2015-03

Roig, R., Mengual-Andrés, S., \& Suárez, C. (2014). Evaluación de la calidad pedagógica de los MOOC. Profesorado. Revista de Currículum y Formación del Profesorado, 18(1), 27-41.

Rosselle, M., Caron, P. A., \& Heutte, J. (2014). A typology and dimensions of a description framework for MOOCs. In U. Cress y C. Delgado (Coords.), EMOOCs 2014. European MOOCs Stakeholders Summit. Proceedings of the European MOOC Stakeholder Summit 2014 (pp. 130139). Recuperado desde http://goo.gl/kzOkLn

Sarabia, M. C. (2016). Nuevas culturas educativas: los MOOCs en las universidades españolas. Cultura y Educación: Revista de teoría, investigación y práctica, 28(1), 204-212.

SCOPEO. (2013). MOOC: Estado de la situación actual, posibilidades, retos y futuro. Informe SCOPEO $n^{o}$ 2. Recuperado desde http://goo.gl/KOQslU

Sharples, M., Mcandrew, P., Weller, M., Ferguson, R., Fitzgerald, E., Hirst, T., . . Whitelock, D. (2012). Innovating Pedagogy 2012. Exploring new forms of teaching, learning and assessment, to guide educators and policy makers. Open University Innovation Report $\mathrm{n}^{\circ} 1$. Milton Keynes: The Open University.

Siemens, G. (2005). Connectivism: A Learning Theory for a Digital Age. International Journal of Instructional Technology and Distance Learning, 2(1), 3-6.

Siemens, G. (2012). MOOCs are really a platform, Elearnspace. Recuperado desde http://goo.gl/ MM5y00

Strijbos, J., Martens, R., \& Jochems, W. (2004). Designing for Interaction: Six Steps to Designing Computer-Supported Group-based Learning. Computers y Education, 42, 403-424. doi:10.1016/j. compedu.2003.10.004

Vázquez-Cano, E. (2013). El videoartículo: nuevo formato de divulgación en revistas científicas y su integración en MOOCs. Comunicar, 41, 83-91. doi:10.3916/C41-2013-08

Vázquez-Cano, E., López, E., \& Sarasola, J. L. (2013). La expansión del conocimiento en abierto: los MOOC. Barcelona: Octaedro. 
Vidal, A., \& Camarena, B. O. (2014). Retos y posibilidades de los cursos en línea a partir de una experiencia concreta. Pixel Bit. Revista de Medios y Educación, 44, 19-34.

Yuan, L., \& Powell, S. (2013). MOOCs and Open Education: Implications for Higher Education. UK: Cetis.

Zapata-Ros, M. (2013a). MOOCs, una visión crítica y una alternativa complementaria: La individualización del aprendizaje y de la ayuda pedagógica. Campus Virtuales, 1(II), 20-38.

Zapata-Ros, M. (2013b). El diseño instruccional de los MOOCs y el de los nuevos cursos online abiertos personalizados (POOCs). Recuperado desde http://eprints.rclis.org/19744/ 